is naturaleza 
Para la publicación de este número se ha contado con la ayuda

financiera de las siguientes instituciones:

Departamento de Filosofía y Lógica y Filosofía de la Ciencia de la Universidad de Sevilla

Facultad de Filosofía de la Universidad de Sevilla 


\section{NATURALEZA Y LIBERTAD}

Revista de estudios interdisciplinares

Número 4

Málaga, 2014

Esta revista es accesible on-line en el siguiente portal:

http://grupo.us.es/naturalezayl 


\section{Naturaleza y Libertad}

Revista de estudios interdisciplinares

Número 4

ISSN: 2254-96682014

Directores: Juan Arana, Universidad de Sevilla; Juan José Padial, Universidad de Málaga; Francisco Rodríguez Valls, Universidad de Sevilla.

Secretaria: Avelina Cecilia Lafuente, Universidad de Sevilla.

Consejo de Redacción: Jesús Fernández Muñoz, Universidad de Sevilla; José Luis González Quirós, Universidad Juan Carlos I, Madrid; Francisco Soler, Universität Dortmund / Universidad de Sevilla; Pedro Jesús Teruel, Universidad de Valencia; Héctor Velázquez, Universidad Panamericana, México.

Adjunto a la redacción: Miguel Palomo, Universidad de Sevilla

Consejo Editorial: Mariano Álvarez, Real Academia de Ciencia Morales y Políticas; Allan Franklin, University of Colorado; Michael Heller, Universidad Pontificia de Cracovia; Manfred Stöcker, Universität Bremen; William Stoeger, University of Arizona.

Consejo Asesor: Rafael Andrés Alemañ Berenguer. Universidad de Alicante; Juan Ramón Álvarez, Universidad de León; Luciano Espinosa, Universidad de Salamanca; Miguel Espinoza, Université de Strasbourg; Juan A. García González, Universidad de Málaga; José Manuel Giménez Amaya, Universidad de Navarra; Karim Gherab Martín, Urbana University, Illinois; Martín López Corredoira, Instituto de Astrofisica de Canarias; Alfredo Marcos, Universidad de Valladolid; Marta Mendonça, Universidade Nova de Lisboa; Javier Monserrat, Universidad Autónoma de Madrid; Leopoldo Prieto, Colegio Mayor San Pablo, Madrid; Ana Rioja, Universidad Complutense, Madrid. Madrid; José Luis González Recio, Universidad Complutense, Madrid; Javier Serrano, TEC Monterrey (México); Hugo Viciana, Université Paris I; Claudia Vanney, Universidad Austral, Buenos Aires; José Domingo Vilaplana, Huelva.

\section{Redacción y Secretaria:}

Naturaleza y Libertad. Revista de estudios interdisciplinares. Departamento de Filosofia y Lógica. Calle Camilo José Cela s.n. E-41018 Sevilla.

Depósito Legal: MA2112-2012

욜 954.55.77.57 Fax: 954.55.16.78. E-mail: jarana@us.es

(C) Naturaleza y Libertad. Revista de Filosofía, 2014 


\section{ÍNDICE}

\section{ESTUDIOS}

Manuel Alfonseca (Universidad Autónoma de Madrid), Diseño inteligente, evolución al azar, o evolución providencial....... ..11

Paul Gilbert, S J (U. Gregoriana, Roma), Deseo de conocer ..............................................27

Javier Hernández-Pacheco (U. de Sevilla), Evolución, erotismo y origen de las especies. De vuelta desde Darwin a Platón y Aristóteles.

Miguel Ángel Herrero (U. Politécnica de Madrid), La formación de los conceptos científicos. De Grosseteste a Galileo.

Ma Teresa Pelacho López (Zaragoza), Otra autonomía de la ciencia. Reflexiones a la luz del pensamiento de Serguei Kara-murzá.......

Juan Fernando Sellés (U. de Navarra), Estudio de las tesis centrales de Millán-Puelles sobre la libertad....... 189

Francisco Soler Gil (U. de Sevilla), El naturalismo y la tentación de las extrapolaciones omnicomprensivas.

Héctor Velázquez Fernández (U. Panamericana, México), En torno a la naturalización de la conciencia sugerida por Daniel Dennett

\section{SECCIÓN BIBLIOGRÁFICA}

Maria Camila Gallego: Falsos saberes.

Juan Arana: Bernardino Telesio, La naturaleza según sus propios principios. 


\title{
DISEÑO INTELIGENTE, EVOLUCIÓN AL AZAR, O EVOLUCIÓN PROVIDENCIAL
}

\author{
Manuel Alfonseca \\ Universidad Autónoma de Madrid
}

\begin{abstract}
Resumen: Como todas las teorías científicas, la teoría de la evolución evoluciona. Sin embargo, eso no quiere decir que esté en crisis, como defienden diversos movimientos creacionistas. En particular, la teoría del diseño inteligente sostiene, apoyándose en argumentos supuestamente científicos, que la evolución no puede tener lugar sin intervención divina. En la postura opuesta, el ateísmo evolucionista sostiene que la evolución es mero resultado del azar, sin participación alguna del diseño. Ambas posturas son extra-científicas, porque no se puede demostrar que sean falsas. A lo sumo, pueden considerarse como hipótesis metafísicas. La evolución providencial, propuesta por el autor apoyándose en sus investigaciones en el campo de la vida artificial, que proporciona un ejemplo de la combinación de azar y diseño, podría considerarse como una versión puramente metafísica del diseño inteligente.
\end{abstract}

Palabras clave: Teoría de la evolución. Diseño inteligente. Evolución providencial. Azar y diseño.

Abstract: As every scientific theory, the theory of evolution evolves, but this does not mean that this theory is suffering a crisis, as upheld by various creationist movements. In particular, the theory of intelligent design asserts, relying on apparently scientific arguments, that evolution cannot take place except through divine intervention. As the opposite stance, evolutionary atheism asserts that evolution is a mere result of chance, without any participation of design. Both stances are extra-scientific, because they are un-falsifiable. At most, they can be considered as metaphysical hypotheses. Providential evolution, proposed by the author relying on his research in the field of artificial life, which provides an example of the combination of chance and design, could be considered as a purely metaphysical version of intelligent design.

Keywords: Evolutionary theory. Intelligent design. Providential evolution. Chance and design.

Recibido: 03/03/2014. Aprobado: 21/03/2014 
Naturaleza y Libertad. Revista de estudios interdisciplinares. Número 4, 2014. ISSN: 2254-9668

\section{LA EVOLUCIÓN DE LA TEORÍA DE LA EVOLUCIÓN}

Como toda teoría científica, la teoría de la evolución siempre será provisional y estará sujeta a correcciones y cambios. Sin embargo, tras un siglo y medio de vigencia, lo esencial de ella puede considerarse definitivamente establecido, gracias a los argumentos proporcionados por diversas disciplinas relacionadas con ella: Anatomía comparada, Embriología, Paleontología, Biogeografía, Biología molecular

Como consecuencia de estos apoyos, resulta muy improbable que la teoría de la evolución sea derrocada en el futuro por nuevos avances de la ciencia. Lo que sí puede ocurrir, sin embargo, es que se vea sometida a modificaciones de detalle, como siempre le ocurre a cualquier teoría científica.

De hecho, esos cambios y avances se han producido ya con cierta frecuencia y más de una vez. En su siglo y medio de historia, la teoría de la evolución ha pasado por las siguientes fases:

En su famosísimo libro ${ }^{1}$, Charles Darwin propuso la selección natural como motor de la evolución, pero no pudo explicar cómo se producían las variaciones aleatorias sobre las que pudiera actuar dicha selección, ni cómo podrían pasar esas variaciones de una generación a la siguiente.

1 On the origin of species by means of natural selection, 1859. 
1. En 1866, Gregor Mendel descubrió las leyes de la herencia, pero como las publicó en una revista poco conocida ${ }^{2}$, pasaron desapercibidas hasta que treinta años más tarde tres investigadores volvieron a descubrirlas ${ }^{3}$, revisaron la literatura científica, dieron con la publicación de Mendel y reconocieron la prioridad de su descubrimiento.

2. Las leyes de Mendel daban respuesta a una de las dos cuestiones pendientes en la teoría de la evolución: la transmisión hereditaria de las diferencias. Quedaba por contestar la primera: ¿cómo surgen características nuevas que antes no existían? La respuesta la obtuvo el holandés Hugo de Vries, quien en 1900 descubrió las mutaciones.

3. Los experimentos del biólogo norteamericano Thomas Hunt Morgan (premio Nobel de Fisiología y Medicina en 1933), realizados en la década de 1910 sobre la mosca de las frutas, Drosophila melanogaster, desarrollaron la naciente ciencia de la Genética y llevaron a la demostración de que las mutaciones no son otra cosa que modificaciones en la estructura de los genes, que a su vez forman parte de los cromosomas de las células vivas, aunque por entonces no se sabía qué sustancia química concreta desempeñaba ese papel.

4. Hacia 1930, un grupo de biólogos encabezados por Theodosius Dobzhansky, Ernst Mayr y George Gaylord Simpson integró estos hechos y teorías científicas en un nuevo cuerpo de doctrina que recibió el nombre de 
Naturaleza y Libertad. Revista de estudios interdisciplinares. Número 4, 2014. ISSN: 2254-9668

neodarwinismo o teoría sintética de la evolución. De acuerdo con esta teoría, la evolución no actúa sobre individuos aislados, sino sobre poblaciones completas. Una población consta de un conjunto de individuos, cada uno de los cuales tiene una dotación genética concreta como resultado de las mutaciones acumuladas por sus antecesores, que se le han transmitido de acuerdo con las leyes de Mendel. En la población total, muchos genes se encuentran representados por dos o más variedades diferentes (alelos). No existe, por tanto, una composición genética única, sino una mezcla de alternativas que se barajan en cada generación como consecuencia de la reproducción sexual.

5. En 1944, Oswald Theodore Avery descubrió que el ADN de los cromosomas es la molécula portadora de la herencia. Y en 1953, James Watson, Francis Crick y Rosalind Franklin desentrañaron la estructura en doble hélice de dicha molécula.

6. En 1972, Stephen Jay Gould y Niles Eldredge propusieron la teoría del equilibrio puntuado (Eldredge \& Gould, 1972), que afirma que la evolución no es un proceso continuo, pues largos periodos de estancamiento pueden ser seguidos por otros más cortos, en los que tienen lugar numerosos cambios.

7. En 1976, y en contraposición a la teoría de Gould y Eldredge, Richard Dawkins propuso la teoría del gen egoísta (Dawkins, 1976), que afirma que los fenotipos de los seres vivos son simples herramientas que utilizan los genes para perpetuarse, y que son estos (los genes) las unidades evolutivas fundamentales. Dawkins y Gould mantuvieron una acalorada polémica res- 
pecto a sus teorías respectivas durante más de 20 años, hasta la muerte del segundo.

8. En 1983, Motoo Kimura propuso la teoría neutralista de la evolución (Kimura, 1983), según la cual la mayor parte de las mutaciones son neutrales respecto a la selección natural y no son afectadas por ella, lo que contribuiría al mantenimiento de una variabilidad genética mucho mayor en las poblaciones. En la actualidad, aunque la teoría neutralista no se acepta tal como la presentó Kimura, sí parece haber consenso respecto a que la evolución se comporta, al menos, como un proceso cuasi-neutralista.

9. A partir del año 2000 han tenido lugar nuevos avances ${ }^{4}$ relacionados con la teoría de la evolución, en campos como la epigenética (que estudia las interacciones entre los genes y algunos casos de herencia no mendeliana) y el desarrollo embrionario asimétrico (la diferente influencia de los genes maternos y paternos durante el desarrollo), entre otros.

\section{QUÉ ES LA TEORÍA DE LA EVOLUCIÓN}

Una vez comprobado que la teoría cientifica de la evolución evoluciona, debemos plantearnos cómo puede definirse. En primer lugar, señalaremos que el uso de la palabra cientifica supone que esta teoría tiene que ver con: a) he-

4 The year in evolutionary biology, Annals of the New York Academy of Sciences, 2008. 
Naturaleza y Libertad. Revista de estudios interdisciplinares. Número 4, 2014. ISSN: 2254-9668

chos verificables; b) hipótesis que los explican, que siempre estarán sujetas a la posibilidad de que al final se pruebe que dichas hipótesis son falsas (Popper, 1934).

En este contexto, la teoría de la evolución se basa en la observación comprobable de que las especies de los seres vivos cambian con el tiempo, y analiza los mecanismos por los que esto puede llegar a ocurrir: $\mathrm{ADN}$, mutaciones, selección natural, etcétera. En su forma actual, suele considerarse que la evolución depende de la interacción de tres factores distintos (Monod, 1970):

1. La variabilidad genética de los seres vivos, que reside principalmente en el ADN, se hereda generalmente según las leyes de Mendel y se modifica aleatoriamente como consecuencia de la acción de los rayos cósmicos y otros agentes mutagénicos, o a través de la recombinación genética y otras modificaciones que pueden tener lugar durante la reproducción sexual.

2. El ambiente, que también cambia de forma aleatoria.

3. La selección natural, que no es otra cosa que la constatación del hecho de que los individuos mejor adaptados al ambiente se reproducen más que los menos adaptados y transmiten sus genes en mayor proporción a la generación siguiente.

Aquí se debería mencionar un cuarto elemento, aunque rara vez se hace: las leyes de la naturaleza (la relatividad general y la física cuántica) y las constantes fundamentales, que parecen ajustadas de forma crítica para hacer 
posible la vida. El problema del ajuste fino constituye un indicio (no una prueba científica) hacia la posibilidad del diseño.

\section{QUÉ ES EL DISEÑO INTELIGENTE}

El creacionismo es el intento del fundamentalismo religioso por presentar la Biblia como fuente de información científica. Esta corriente se da especialmente entre ciertos grupos protestantes norteamericanos, aunque en su forma más moderna se ha extendido un poco a algunos católicos y al otro lado del Atlántico.

La actividad de los creacionistas dura ya casi un siglo. En 1925, se aprobó en el estado de Tennessee una ley que declaraba «ilegal que ningún profesor de cualquiera de las universidades, escuelas normales y todas las demás escuelas públicas del estado (que son financiadas en su totalidad o en parte por los fondos para la enseñanza pública del Estado), impartan cualquier teoría que niegue la historia de la Creación divina del hombre tal como la enseña la Biblia, y digan, en su lugar, que el hombre desciende de un orden inferior de animales» ${ }^{5}$. Para echarla abajo, los contrarios a la ley montaron el famoso juicio contra John Scopes (un profesor de educación física que se ofreció voluntario para hacer de chivo expiatorio), aunque su intento no dio resultado, pues el juicio fue anulado sin entrar en el fondo del asunto, porque el

5 Citada en Gould, 1983. 
Naturaleza y Libertad. Revista de estudios interdisciplinares. Número 4, 2014. ISSN: 2254-9668

juez se extralimitó al imponer al acusado una multa de 100 dólares. Una versión algo tergiversada del juicio aparece en la película de Stanley Kramer Inherit the wind, de 1960.

A partir de ahí, el creacionismo ha realizado tres intentos sucesivos para influir en la enseñanza norteamericana:

1. El primer paso fue intentar que se aprobasen leyes que prohibiesen la enseñanza de la teoría de la evolución en las escuelas. Su mayor éxito fue la aprobación en 1928 del Estatuto de Arkansas, que fue revocado por el Tribunal Supremo en 1968.

2. El segundo paso (el tratamiento equilibrado) trataba de conseguir que se enseñase el creacionismo bíblico (basado en la interpretación literal de los primeros capítulos del Génesis) como alternativa a la teoría de la evolución en las clases de biología. Lo consiguieron en 1981 en el estado de Luisiana, pero esta decisión fue revocada por el Tribunal Supremo en 1987.

3. El tercer intento se conoce con el nombre (poco apropiado, pero lamentablemente ha cuajado) de diseño inteligente, que acepta la evolución, pero sostiene que es posible demostrar científicamente que estos procesos no siempre se basan en el azar, sino que a veces requieren la intervención directa de un ser divino. Los intentos de que la legislación obligue a enseñar esta teoría cientifica en las escuelas han pasado ya por los tribunales y probablemente acabarán por forzar una decisión definitiva del Tribunal Supremo, que previsiblemente volverá a ser negativa. 
El diseño inteligente, tal como lo definen estos nuevos creacionistas, afirma que en los seres vivos existen casos de complejidad irreducible (Behe, 1996) que es imposible explicar como efecto de la casualidad. Para probarlo, proponen diversos ejemplos, como los siguientes: A) La estructura compleja del ojo de los vertebrados. B) Los flagelos rotatorios de ciertas bacterias. C) La conducta de los himenópteros cazadores, que paralizan sus presas inyectando veneno en cada uno de sus ganglios nerviosos (hasta en nueve sitios diferentes). D) El ciclo de Krebs, que permite a los seres vivos aerobios respirar oxígeno, a través de un proceso complejo en el que participan numerosos enzimas que actúan de forma interconectada.

Estos argumentos se presentan a veces como si fuesen nuevos e incontestables, cuando algunos se remontan al propio Darwin, que en el Origen de las Especies ofreció una explicación al proceso de evolución del ojo, o al naturalista francés Fabre ${ }^{6}$, que en el siglo XIX descubrió el comportamiento de los himenópteros cazadores, utilizado a principios del siglo XX por Henri Bergson ${ }^{7}$ en el contexto de su teoría de l'elan vital ${ }^{8}$.

6 J. H. Fabre, Nouveaux souvenirs entomologiques, 1882.

7 H. Bergson, L'evolution creatrice, 1907.

8 Es curioso que fuese el propio Fabre quien, en la misma obra citada, describió otra especie de himenóptero cuya conducta podría servir de paso intermedio (eslabón perdido) para la aparición de la conducta más compleja de los himenópteros cazadores. 
Naturaleza y Libertad. Revista de estudios interdisciplinares. Número 4, 2014. ISSN: 2254-9668

\section{DiSEÑO INTELIGENTE O EVOLUCIÓN AL AZAR}

Cuando el diseño inteligente se presenta como una teoría científica, suele causar la indignación de muchos otros científicos, que no aceptan tal equiparación y lo consideran un intento de introducir una teoría filosófica o religiosa como alternativa seudocientífica a la teoría de la evolución. Lo malo es que algunos científicos caen en el mismo defecto que critican y presentan especulaciones filosóficas y afirmaciones dogmáticas como si fuesen teorías científicas verificables. Presentar como científica cualquiera de las dos afirmaciones siguientes es igualmente censurable, porque ninguna de las dos lo es: A) Detrás de la evolución de los seres vivos hay un diseño inteligente. B) No existe diseño. La evolución de los seres vivos es resultado del puro azar.

Es evidente que ambas posturas son irreconciliables. Por otra parte, tampoco es previsible que una de ellas consiga imponerse a la otra por procedimientos estrictamente científicos. Supongamos que se detectara en los seres vivos algún fenómeno que sea difícil de explicar como efecto de la casualidad, como alguno de los casos de complejidad irreductible que presenta Behe. En tal caso, un ateo siempre podrá afirmar que esos casos serán explicados satisfactoriamente en el futuro, apoyándose en la experiencia pasada de ejemplos similares, como alguno de los mencionados más arriba. De paso, acusará al defensor del diseño inteligente de creer en un Dios de los huecos. Por otra parte, aunque todo lo que vemos en los seres vivos fuese compatible con el puro azar, la hipótesis filosófica del diseño inteligente no podría que- 
Naturaleza y Libertad. Revista de estudios interdisciplinares. Número 4, 2014. ISSN: 2254-9668

dar excluida, pues no hay nada que impida, en principio, que Dios haya incluido el azar (o algo parecido a este) entre las herramientas con las que ha creado el universo. ¿O acaso vamos a negarle a Dios la capacidad de utilizar mecanismos que nosotros mismos usamos?

$\mathrm{Ni}$ el diseño inteligente ni la evolución debida únicamente al azar son teorías científicas, porque es imposible probar que son falsas. Las dos pertenecen al campo de la filosofía y deben presentarse como tales. Está fuera de lugar que los libros de texto sobre biología presenten el diseño inteligente como alternativa a la teoría de la evolución, porque no lo es. Pero tampoco deben afirmar cosas como estas: la ciencia ha demostrado que Dios no existe; o la ciencia ha demostrado que el universo (o la vida) es resultado del puro azar; porque ambas afirmaciones son falsas. La ciencia no puede demostrarlas.

\section{INVESTIGACIÓN SOBRE LA VIDA ARTIFICIAL}

Hay indicios recientes que apuntan que evolución y diseño son perfectamente compatibles. Existe un campo de la informática (la programación evolutiva) que se inspira en la evolución biológica para diseñar programas de ordenador. Una de sus ramas, la investigación sobre vida artificial, tiene por objeto el estudio de agentes cuyo comportamiento recuerda el de los seres vivos. El autor de este artículo ha trabajado en varias de las líneas más frecuentes, como la simulación de colonias de hormigas (Alfonseca \& De Lara, 2002), que arroja luz sobre la conducta de enjambres de seres que actúan 
juntos, y permite formular hipótesis sobre la emergencia de entidades de orden superior: organismos pluricelulares, sociedades de insectos, o incluso la sociedad humana; ecologías artificiales (Alfonseca \& Soler Gil, 2013), que permiten analizar la interacción de varias especies a lo largo del proceso evolutivo; analizadores de la evolución de lenguajes simplificados en poblaciones de seres simulados; o sistemas muy sencillos, a los que se deja evolucionar libremente y en los que aparecen fenómenos como parásitos, parásitos de parásitos, etcétera (Ray, 1990).

Cuando se diseña un experimento de vida artificial, hay que considerar cuatro factores:

1. Variaciones aleatorias (mutaciones y recombinaciones, entre otras) en los genomas de los seres vivos simulados. Estas variaciones no son realmente aleatorias, sino seudoaleatorias, pues se emplean algoritmos para generarlas.

2. El ambiente, que también puede cambiar de modo seudoaleatorio.

3. La selección natural, que favorece la supervivencia de los genomas que mejor se adaptan al ambiente, a través del cálculo de una función de adecuación (fitness) no aleatoria.

4. Las reglas básicas del juego: las instrucciones que pueden combinar los seres vivos del experimento pata llevar a cabo su evolución, y que afectan a su comportamiento.

Se observará que los experimentos sobre vida artificial son muy semejantes a la evolución biológica. Sin embargo, los primeros ofrecen un ejemplo 
Naturaleza y Libertad. Revista de estudios interdisciplinares. Número 4, 2014. ISSN: 2254-9668

claro de diseño por parte del programador. Si algún día mis experimentos avanzaran tanto que llegaran a surgir en ellos seres inteligentes, dichos seres no serían capaces de deducir mi existencia realizando experimentos dentro de su mundo, porque yo no pertenezco a ese mundo. Quizá se les ocurrirá aplicar el principio fundamental del evolucionismo filosófico ateo, que puede resumirse en el siguiente razonamiento:

En todos los procesos evolutivos intervienen el azar y la selección natural.

Luego es probable que Dios no exista.

Esto les llevaría a formular el siguiente razonamiento paralelo:

En todos mis experimentos de vida artificial intervienen el pseudoazar y la selección natural.

Luego es probable que yo no exista.

Es evidente, sin embargo, que en el segundo caso la conclusión es falsa, lo que debería hacernos recapacitar que muy bien podría serlo también la primera.

Pero ¡un momento! ¿no hay una diferencia importante entre los dos razonamientos, que puede echar por tierra el paralelismo? Donde en el primero dice azar, en el segundo dice pseudoazar. ¿No podrían los hipotéticos seres inteligentes que puedan llegar a aparecer en mis experimentos deducir mi 
Naturaleza y Libertad. Revista de estudios interdisciplinares. Número 4, 2014. ISSN: 2254-9668

existencia demostrando que el supuesto azar que parece regir sus vidas no es realmente azar, sino pseudoazar, lo que les demostraría mi existencia?

Existe un teorema matemático (Chaitin, 1975) que demuestra que, si tenemos una sucesión de números enteros suficientemente compleja, de origen desconocido, es imposible distinguir si dicha sucesión se ha originado aleatoriamente, o si es el resultado de la aplicación de un algoritmo (es pseudoaleatoria). Por lo tanto, mis hipotéticos seres inteligentes no serían capaces de detectar mis algoritmos, y no podrán demostrar mi existencia, igual que nosotros mismos no podemos demostrar que el azar aparente del universo no sea un seudoazar generado algorítmicamente por Dios ${ }^{9}$.

Luego los dos razonamientos descritos más arriba son indistinguibles. Si mis seres hipotéticos llegasen a la conclusión de que yo no existo, de que su mundo surgió por casualidad, estarían equivocados, pues sabemos que ese mundo fue diseñado por mí.

\section{EVOLUCIÓN PROVIDENCIAL}

Ante el descrédito en que ha caído el término diseño inteligente, a consecuencia del intento de hacerlo pasar por una teoría científica, prefiero utilizar

9 La importancia de este teorema en este contexto fue señalada por Fernando Sols en su artículo «Heisenberg, Gödel y la cuestión de la finalidad en la ciencia», Emilio Chuvieco (ed), Fundación Ramón Areces, Madrid, 2012. 
Naturaleza y Libertad. Revista de estudios interdisciplinares. Número 4, 2014. ISSN: 2254-9668

el nombre de evolución providencial para referirme a la teoría filosófica que afirma que el azar que subyace a la evolución puede ser realmente seudoazar, y que nuestro universo y la vida en la Tierra han sido diseñados por Dios. Científicamente, como indica el teorema de Chaitin, esto no se puede demostrar, como tampoco puede demostrarse la hipótesis atea contraria, que sostiene que todo es puro azar. La cuestión resulta así ser extra-científica, pero el hecho de que dispongamos de un paralelo muy sencillo (la vida artificial) en el que el argumento ateo resulta claramente falso, debería servir de indicio y hacernos pensar.

\section{BIBLIOGRAFÍA EMPLEADA}

M. Alfonseca, J. de Lara, "Two level evolution of foraging agent communities" en: BioSystems, 2002 (66, 1-2), pp. 21-30.

M. Alfonseca, F.J. Soler Gil, "Evolving an Ecology of Mathematical Expressions with Grammatical Evolution” en: BioSystems, 2013 (111, 2), pp. 111-119.

M. J. Behe, Darwin's black box, Maine, The Free Press, 1996.

G. Chaitin, "Randomness and Mathematical Proof", en: Scientific American, 1975 (232, 5), pp. 47-52, http://www.cs.auckland.ac.nz/ chaitin/sciamer.html.

R. Dawkins, The selfish gene, Oxford, Oxford University Press, 1976.

N. Eldredge \& S. J. Gould., "Punctuated equilibria: an alternative to phyletic gradualism". en: Th.J.M. Schopf, (ed.), Models in paleobiology, San Francisco, Freeman Cooper and Co., 1972, pp. 82-115.

S. J. Gould, Hen's teeth and horses's toes, New York, Norton, 1983. 
Naturaleza y Libertad. Revista de estudios interdisciplinares. Número 4, 2014. ISSN: 2254-9668

M. Kimura, The Neutral Theory of Molecular Evolution, Cambridge, Cambridge University Press, 1983.

J. Monod, Le hasard et la nécessité, Paris, Éditions du Seuil, 1970.

K. Popper, The logic of scientific discovery, 1934. Versión española en Madrid, Tecnos, 1962.

T. S. Ray, "Evolution and optimization of digital organisms", en: K. R. Billingsley et al. (ed.), Scientific Excellence in Supercomputing: The IBM 1990 Contest Prize Papers, Athens GA, The Baldwin Press, 1991, pp. 489-531.

The year in evolutionary biology, Annals of the New York Academy of Sciences, 2008.

Manuel Alfonseca

Manuel.Alfonseca@uam.es 